\title{
The DECAF Score is a Superior Predictor of In-hospital Death than the BAP-65 Score
}

\section{Echevarria $\mathrm{C}^{1,2 *}$, Steer $\mathrm{J}^{1}$ and Bourke $\mathrm{SC}^{1,2}$}

${ }^{1}$ Northumbria Healthcare NHS Foundation Trust, Respiratory Medicine Newcastle upon Tyne, UK

${ }^{2}$ nstitute of Cellular Medicine, Newcastle University, Newcastle Upon Tyne, UK

Tabet and others describe the use of the BAP-65 score to predict mortality and the need for mechanical ventilation in 980 patients admitted with an AECOPD to two Lebanese hospitals over an eightyear period [1]. In their introduction they state that the use of riskstratification tools has not been recommended in practice in patients with AECOPD. However, in the British Thoracic Society COPD audit report, it is recommended that the DECAF score is documented routinely in all patients admitted with an AECOPD [2].

Tabet and others recommend the use of BAP-65 over DECAF, as scoring of BAP-65 is "consistent and generalisable". However, DECAF showed consistent and excellent discrimination in the derivation and validation studies (area under the receiver operator characteristic (AUROC) curve 0.86 and 0.83 respectively) $[3,4]$ and outperformed BAP-65, which performed inconsistently (AUROC 0.65 and 0.77 respectively). One potential weakness of BAP-65 is that the assessment of mental status may vary, despite their claim that the BAP-65 indices are objective. Furthermore, the provision of salbutamol nebulisers by paramedics prior to admission can elevate pulse rate, and reduce its prognostic power. In the DECAF derivation study [3], pulse rate was not associated with in-hospital mortality.

The authors raise concerns regarding the costs of performing investigations to calculate the DECAF score. In common with the GOLD COPD guidelines, we would recommend that chest radiograph, electrocardiogram, full blood count and arterial blood sampling are performed in the assessment of this population [5].
Finally, the authors also state that there are no precise risk stratification tools to help clinicians assess the severity of an AECOPD. In fact, there are multiple tools that perform well in this population, though DECAF has been shown to be superior to alternatives [4]. This is important to acknowledge, as readers are left with the impression that clinicians must rely on judgment alone, which is less accurate than using clinical tools [6].

\section{References}

1. Tabet R, Ardo C, Makrlouf P, Hosry J (2016) Application of Bap-65: A New Score for Risk Stratification in Acute Exacerbation of Chronic Obstructive Pulmonary Disease. J Clin Respir Dis Care 2: 1.

2. Stone RA, Holzhauer-Barrie J, Lowe D (2016) COPD: Who cares matters National Chronic Obstructive Pulmonary Disease (COPD) Audit Programme: Clinical audit of COPD exacerbations admitted to acute units in England and Wales 2014

3. Steer J, Gibson J, Bourke SC (2012)The DECAF Score: predicting hospital mortality in exacerbations of chronic obstructive pulmonary disease. Thorax 67: $970-976$.

4. Echevarria C, Steer J, Heslop-Marshall K, Stenton SC, Hickey PM, et al. (2016) Validation of the DECAF score to predict hospital mortality in acute exacerbations of COPD. Thorax 71: 133-140.

5. Vestbo J, Hurd SS, Agustí AG, Jones PW, Vogelmeier C, et al. (2013) Global strategy for the diagnosis, management, and prevention of chronic obstructive pulmonary disease: GOLD executive summary. Am J Respir Crit Care Med 187: $347-365$

6. Wildman MJ, Sanderson C, Groves J, Reeves BC, Ayres J, et al. (2009) Predicting mortality for patients with exacerbations of COPD and Asthma in the COPD and Asthma Outcome Study (CAOS). QJM 102: 389-399.
*Corresponding author: Carlos Echevarria, Northumbria Healthcare NHS Foundation Trust, Respiratory Medicine, Newcastle upon Tyne, United Kingdom, Tel: +44 191293 4026; Fax: +44 191293 4026; E-mail: CarlosEchevarria@doctors.org.uk

Received April 22, 2016; Accepted May 05, 2016; Published May 09, 2016

Citation: Echevarria C, Steer J, Bourke SC (2016) The DECAF Score is a Superior Predictor of In-hospital Death than the BAP-65 Score. J Clin Respir Dis Care 2: 111. doi: $10.4172 / 2472-1247.1000111$

Copyright: (C) 2016 Echevarria C, et al. This is an open-access article distributed under the terms of the Creative Commons Attribution License, which permits unrestricted use, distribution, and reproduction in any medium, provided the original author and source are credited. 